\title{
Ankylosing spondylitis in West Africans-evidence for a non-HLA-B27 protective effect
}

\author{
M A Brown, A Jepson, A Young, H C Whittle, B M Greenwood, B P Wordsworth
}

Wellcome Trust Centre for Human Genetics, Oxford, United

Kingdom

M A Brown

A Jepson

A Young

B P Wordsworth

MRC Laboratories, Fajara, The Gambia H C Whittle

B M Greenwood

Correspondence to:

Dr M A Brown, Wellcome Trust Centre for Human Genetics, Windmill Road, Headington, Oxford, OX3 $7 \mathrm{BN}$

Accepted for publication 20 September 1996

\begin{abstract}
Objective-To determine the prevalence of ankylosing spondylitis in the Fula ethnic group in The Gambia, and relate the disease prevalence to the B27 frequency and subtype distribution of that population.

Methods-215 first degree relatives of 48 B27 positive Fula twin pairs, and 900 adult Fula males were screened for ankylosing spondylitis by clinical and, where appropriate, radiographic means. The B27 prevalence was determined by PCR/sequence specific oligonucleotides on finger prick samples from 100 unrelated Fula, and B27 subtype distribution by SSCP on unrelated B27 positive individuals. This data were then compared with the prevalence of ankylosing spondylitis among B27 positive Caucasians.
\end{abstract}

Results-No case of ankylosing spondylitis was seen. Six per cent of Fula are B27 positive, of which $32 \%$ are $B \star 2703$ and $68 \%$ $B \star 2705$. Assuming the penetrance of ankylosing spondylitis in B27 positive Fula is the same as in B27 positive Caucasians, the probability of not observing any cases of ankylosing spondylitis among the Fula examined is remote $\left(P=6.7 \times 10^{-6}\right)$. Similarly, the chance of not seeing any cases among those expected to be either $B \star 2705$ or $B \star 2703$ was small $\left(P=3.2 \times 10^{-4}\right.$ for $B \star 2705$, and $P=0.02$ for $B \star 2703$ ).

Conclusions-The risk of developing ankylosing spondylitis in B27 positive Fula is lower than in B27 positive Caucasians. This is not explained by the B27 subtype distribution among Fula, and suggests the presence of some non-B27 protective factor reducing the prevalence of ankylosing spondylitis in this population.

(Ann Rheum Dis 1997;56:68-70)

The exceptionally strong association between B27 and ankylosing spondylitis has been known since 1973. It is remarkably consistent across a wide range of ethnic groups in Europe, the Americas, and Asia (relative risk >100) and the prevalence of ankylosing spondylitis closely parallels the frequency of B27 in most populations that have been studied. ${ }^{1}$ It is therefore very likely that B27 itself, rather than a closely linked gene, is involved in susceptibility to ankylosing spondylitis, probably by its function as a restriction element in the initiation of specific immune responses. ${ }^{2}$ However, there are some exceptions to this general rule which may have important implications for our understanding of the aetiology of this disease. For example, ankylosing spondylitis appears to be extremely rare in The Gambia, as in other parts of tropical Africa, although B27 is actually present at approximately half the frequency of most European populations. ${ }^{3}$ This apparent discrepancy could be related in some way to allelic polymorphism of B27 since this serological specificity is encoded by at least nine different alleles (HLA-B $\left.{ }^{\star} 2701-2709\right) .{ }^{1}$ $B \star 2703$ differs from the predominant Caucasian subtype, $B^{\star} 2705$, only by the substitution of histidine for tyrosine at position 59 , affecting the " $\mathrm{A}$ " peptide binding pocket. There is some evidence that this may affect the peptide binding motif. ${ }^{4}$ The $\mathrm{B}^{\star} 2703$ allele is relatively common in The Gambia ${ }^{3}$ but no examples of patients with ankylosing spondylitis with this subtype have yet been published from The Gambia or elsewhere in the world. It has been suggested that the relevant pathogenic antigens causing ankylosing spondylitis may be bound only by a subset of B27 alleles which does not include $B^{\star} 2703$. Some further support for this hypothesis comes from Thailand where another allele (HLA-B ${ }^{\star} 2706$ ) appears not to be associated with ankylosing spondylitis. $^{5}$

Any conclusions about the susceptibility to ankylosing spondylitis associated with B27 and its subtypes from population studies depends crucially upon accurate ascertainment of cases, as well as on a knowledge of the B27 frequencies in the study populations. The Fula ethnic group in The Gambia provides a potentially valuable study population because of its relatively high frequency $(7.8 \%)$ of $\mathrm{B} 27^{6}$ and potentially high risk of ankylosing spondylitis. Although the Fula could not be described as a genetically isolated population, relatively little intermarriage between the major Gambian ethnic groups is thought to occur, and the B27 frequency in non-Fula Gambians is significantly lower than in Fula themselves. ${ }^{6}$

Rigorous surveys of the prevalence of ankylosing spondylitis have not yet been undertaken in The Gambia, although clinical experience suggests that the disease is rare, consistent with surveys in neighbouring countries. We have therefore determined the frequency of ankylosing spondylitis in a Fula population previously identified as having a relatively high frequency of B27, and related this to the B27 subtype distribution in this ethnic group. 


\section{Methods}

SUBJECTS

Subjects were recruited by two means. First, they were identified from a database established in 1991 during the course of two studies of malaria in twins ( ${ }^{7}$, and Jepson A, et $a l$, unpublished data). Of 480 twin pairs for which HLA class I haplotypes were documented, 39 were identified in which at least one member of the twin pair was B27 positive. Two hundred and fifteen first degree relatives of these twins over the age of 25 years, resident in the Upper River Division of The Gambia, were ascertained and invited to participate in this study. Ethical approval for the study had been obtained from the Gambia Government/MRC joint ethics committee. Second, 900 Fula males ("sporadic males") over the age of 25 years were recruited for clinical examination. Blood samples for HLA typing were obtained by finger prick in 100 of these latter subjects, who were randomly selected and unrelated. Ankylosing spondylitis was defined by the European Spondylarthropathy Study Group Criteria. ${ }^{8}$ A detailed history of back pain, stiffness and peripheral arthritis was taken, lumbar spine movements were assessed (using the modified Schober technique and lateral lumbar flexion), and all other symptomatic areas were examined. Radiographs of the sacroiliac joints and an AP view of the lumbosacral spine were obtained in cases with a history suggestive of inflammatory back pain with restriction of lumbar spine movement (either Schober test $\leq 21 \mathrm{~cm}$, or lateral flexion $\leq 10 \mathrm{~cm}$ ). Inflammatory type back pain was defined by insidious onset, duration $>$ one month, improvement with exercise, association with morning stiffness, and age of onset $<40$ years.

B27 TYPING

DNA from finger prick samples stored on Guthrie cards was extracted by boiling in sodium hydroxide and neutralisation with acetic acid. ${ }^{9}$ DNA was also extracted using Nucleon kits from blood samples taken as part of the earlier twin studies on 42 individuals who were either the parents of the B27 positive twins, or the twins themselves. B27 typing and subtyping were then performed by PCR/ sequence specific oligonucleotides and SSCP as we have previously described..$^{10}$

STATISTICAL METHODS

The objective of this analysis was to determine the probability of us not seeing any cases of ankylosing spondylitis among the sporadic males and family members who were examined, under given assumptions about the penetrances for ankylosing spondylitis in B27 positive individuals. Firstly for a set of $\boldsymbol{M}$ unrelated individuals, each of whom has probability $\boldsymbol{f}$ of carrying the B27 genotype, the total probability $\boldsymbol{P}$ of seeing no occurrences of ankylosing spondylitis is given by

$$
P=\sum_{i=0}^{M} \frac{M ! f^{i}(1-f)^{M-i} p^{i} q^{M-i}}{(M-i) ! i !}
$$

where $\boldsymbol{p}$ is the assumed observational penetrance of the B27 genotype (that is, the chance that a B27 positive subject manifests ankylosing spondylitis and is correctly observed) and $\boldsymbol{q}$ is the equivalent penetrance for subjects who do not have the B27 genotype. Using the values $\boldsymbol{N}=900, \boldsymbol{f}=0.06, \mathbf{p}=0.111$, and $\boldsymbol{q}=0$ in equation (1) gives a probability $\mathrm{P}<$ 0.0025 of our observing no affected individuals among the 900 unrelated males examined.

The calculation is more complex for the members of the family pedigrees since the observed genotype/phenotype information from each individual affects the probability of their relatives having other phenotype/ genotype combinations. In such case the probability of observing a specific set of data phenotypes given B27 status information ("tests") on some individuals is given by:

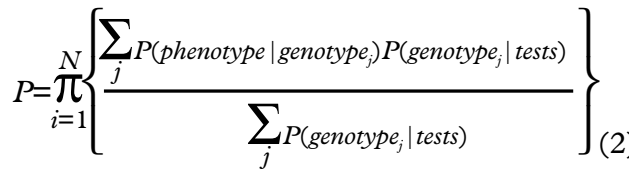

where $\boldsymbol{N}$ is the number of independent family groups in the sample, and the $\boldsymbol{j}$-sum is carried out over all possible genotypic combinations of the $i$ th family group (for the largest family this required evaluating $10^{7}$ terms). Since there were zero affected subjects, $\boldsymbol{P}$ is equal to a one sided $P$ value for the null hypothesis that the postulated disease mechanism is correct, versus the alternate hypothesis that B27 is not causative.

The total $\mathrm{P}$ value for the combined population is given by multiplying the values of equations (1) and (2). To calculate $P$ values for each B27 subtype, the penetrance was reduced according to the proportion of B27 alleles in the population carrying each individual subtype.

The frequency of ankylosing spondylitis in B27 positive individuals varies considerably between surveys. We have used the results of the survey by Gran et al of 14539 Norwegians. ${ }^{11}$ This survey was chosen because of its large sample size, and use of both clinical and radiographic screening for ankylosing spondylitis. It indicates that among B27 positive individuals, $11.1 \%$ of males and $1.5 \%$ of females develop ankylosing spondylitis.

\section{Results}

A total of 215 family members of B27 positive twins and 900 random Fula males were seen and examined. The mean age (SE) of the family members was 47 (15) years and the random Fula males 43 (15) years. The mean age [46 (14)] years and frequency of inflammatory back pain $(7 \%)$ among the 100 randomly selected Fula from whom finger prick samples were taken did not differ from that of the overall study population. The mean age of our study group was slightly older than that in Gran's survey ${ }^{11}$ (mean age 36.5 years). Penetrance of ankylosing spondylitis in our older population would be expected to have been slightly higher than observed by Gran et al, making our subsequent conclusions more 
conservative. The B27 phenotype frequency among these subjects was $6 \%$, and the calculated allele frequency $3 \%$. In all, 21 B27 positive unrelated Fula were available for subtyping (six from the sporadic males and 15 from the $B 27$ positive twins): six were $B^{\star} 2703$ and 15 were $B^{\star} 2705$. Pooling our results with those of Fula previously reported $\left(2 / 4 B^{\star} 2703\right.$, $2 / 4 \mathrm{~B}^{\star} 2705-$ Hill A V S, personal communication) suggests that $32 \%$ of $\mathrm{B} 27$ alleles are $\mathrm{B}^{\star} 2703$ and $68 \% \mathrm{~B}^{\star} 2705$.

Forty seven individuals reported inflammatory low back pain. Four of these had limitation of lumbar spine movement (one female, three males); none had significant arthritis elsewhere. Radiographs of the sacroiliac joints and lumbosacral spine in these cases were normal. Twenty five individuals had thoracic spine pain, and five had cervical spine pain. In all cases the range of motion was normal. Thus no case of ankylosing spondylitis was seen during the survey, consistent with previous reports from nearby countries. No case of reactive arthritis was seen either.

Assuming the same risk for ankylosing spondylitis among B27 positive Gambians as among B27 positive Caucasians, the chance that no case of ankylosing spondylitis would be found among the Gambian population we studied is remote $\left(\mathrm{P}=6.7 \times 10^{-6}\right)$. This finding remains significant even if the risk for ankylosing spondylitis among B27 positive Caucasians was as low as $2.7 \%$ in males and $1 \%$ in females, or if the sensitivity of our screening was as low as $24 \%$. Similarly, the chance of not seeing any cases among those expected to be either $\mathrm{B}^{\star} 2705$ or $\mathrm{B}^{\star} 2703$ was small $\left(\mathrm{P}=3.2 \times 10^{-4}\right.$ for $\mathrm{B}^{\star} 2705$, and $\mathrm{P}=0.02$ for $\mathrm{B}^{\star} 2703$ ).

\section{Discussion}

Our study has confirmed that ankylosing spondylitis is rare among the Fula population in The Gambia, even among those carrying HLA-B27. It has been suggested that the rarity of ankylosing spondylitis in sub-Saharan Africa is due to the low prevalence of B27, or to a lower risk of ankylosing spondylitis in individuals carrying $B^{\star} 2703$ compared with ankylosing spondylitis associated subtypes. ${ }^{3}$ However, neither hypothesis adequately explains the situation in The Gambia: B27 is nearly as common among Fula as it is in most Caucasian populations, and ankylosing spondylitis was found in neither $B^{\star} 2703$ nor $B^{\star} 2705$ individuals. This observation could be explained by the absence of the environmental trigger for ankylosing spondylitis or, perhaps, by the absence of additional (non-B27) genetic susceptibility factors from this population. Alternatively there may be protective environmental or genetic factors operating. A previous survey of B27 positive American Africans found the relative risk of ankylosing spondylitis to be lower than in B27 positive Caucasians, ${ }^{12}$ supporting the theory that some races may be genetically protected from spondyloarthritis. Although we and others have found no evidence for differential susceptibility to ankylosing spondylitis between the two common subtypes $\left(B^{\star} 2705\right.$ and $\left.B \star 2702\right)$ in Caucasians ${ }^{1013}$ the possibility that not all of the various B27 alleles are equally associated with ankylosing spondylitis has been suggested by two previous studies. ${ }^{35}$ The present study strongly suggests that neither $\mathrm{B}^{\star} 2705$ nor $\mathrm{B}^{\star} 2703$ is associated with ankylosing spondylitis in The Gambia. Further studies of West Africans living abroad may help to evaluate the relative importance of environmental and genetic effects (including the role of $\mathrm{B}^{\star} 2703$ ) in the development of ankylosing spondylitis.

We thank the subjects who participated in this study, and also Sheriff Bah, Dawda Sinera, and Galo Touray for their assistance in the field. Fatou Sisay-Joof carried out the HLA typing of the original twin pairs. Angela Frodsham assisted by carrying out the DNA extraction of the twin and twin parent samples. MAB was supported by the Arthritis and Rheumatism Council. This study was made possible by a financial grant from the University of Oxford.

1 Khan M. HLA-B27 and its subtypes in world populations. Curr Opin Rheumatol 1995;7:263-9.

2 McMichael A, Bell J. HLA B27: a disease-associated mmune response gene. Res Immunol 1991;142:475-82.

3 Hill AV, Allsopp CE, Kwiatkowski D, Anstey NM, Greenwood BM, McMichael AJ. HLA class I typing by PCR: HLA-B27 and an African B27 subtype [see comments]. Lancet 1991:337:640-2.

4 Colbert RA, Rowland-Jones SL, McMichael AJ, Frelinger JA. Differences in peptide presentation between B27 subtypes: the importance of the P1 side chain in maintaining high affinity peptide binding to $\mathrm{B}^{\star} 2703$. Immunity high affinity

5 Lopez-Larrea C, Sujirachato K, Mehra NK, Chiewslip P, Isarangkura D, Kanga U, et al. HLA-B27 subtypes in Asian patients with ankylosing spondylitis. Evidence for new associations. Tissue Antigens 1995;45:169-76.

6 Allsopp CE, Harding RM, Taylor C, Bunce M, Kwaitowski $\mathrm{D}$, Anstey $\mathrm{N}$, et al. Interethnic genetic differentiation in Africa: HLA class I antigens in The Gambia. Am J Hum Genet 1992;50:411-21.

7 Jepson AP, Banya WA, Sisay-Joof F, Hassan-King M, Bennett S, Whittle HC: Genetic regulation of fever in Plasmodium falciparum malaria in Gambian twin children. J Infect Dis. 1995;172:316-9.

8 Dougados M, van der Linden S, Juhlin F, Huitfield B, Amor B, Calin A, et al. The European Spondylarthropathy Study Group preliminary criteria for the classification of spondylarthropathy. Arthritis Rheum 1991;34:1218-27.

9 Iitia A, Hogdall E, Dahlen P, Huskainen P, Vuust J, Siitari H. Detection of mutation delta F508 in the cystic fibrosis $\mathrm{H}$. Detection of mutation delta F508 in the cystic fibrosis
gene using allele-specific PCR primers and time-resolved gene using allele-specific PCR primers and time
fluorimetry. PCR Methods Appl 1992:157-62.

10 Brown MA, Pile KD, Kennedy LG, Calin A, Darke C, Bell $\mathrm{J}$, et al. HLA class I associations of ankylosing spondylitis in the white population in the United Kingdom. Ann Rheum Dis 1996;55:268-70

11 Gran J, Husby G, Hordvik M. Prevalence of ankylosing spondylitis in males and females in a young middle aged population in Tromso, northern Norway. Ann Rheum Dis 1985;44:359-67.

12 Khan M, Braun W, Jushner I, Grecek DE, Muir WA, Steiberg AG. HLA B27 in ankylosing spondylitis: differences in frequency and relative risk in American blacks and caucasians. J Rheumatol 1977;4(suppl 3):39-43.

13 Breur-Vriesendorp BS, Dekker-Saeys AJ, Ivanyi P. Distribution of HLA-B27 subtypes in patients with ankylosing spondylitis: the disease is associated with a common determinant of the various B27 molecules. Ann Rheum Dis 1987;46:353-6. 